\title{
A CFD study on design parameters acting in cavitation of positive displacement pump
}

\author{
A. Iannetti, M. T. Stickland and W. M. Dempster \\ University of Strathclyde, Mechanical and aerospace engineering department, Glasgow, UK
}

\section{ABSTRACT}

A CFD test case of a positive displacement reciprocating pump is presented to demonstrate the capability and benefits which numerical analysis may bring to designers in terms of information on fluid dynamic fields useful to optimize the geometry of the discussed device in all the different operating conditions; even in the worst operating conditions when cavitation appears. The paper discusses the role of design parameters such as the inlet valve shape, mass and spring preload in full cavitating conditions. The comprehensive CFD model makes use of the Singhal et al.(1) cavitation algorithm in conjunction with an Eulerian multiphase model. User defined functions add a few more functionalities to the CFD solver such as the valve dynamics model and the compressibility of water. For each of the cases, the work presented shows the capability of the CFD technique to predict quantitative results such as the volumetric efficiency loss and amount of water vapour generated when cavitation arises. Providing pump designers with this information before the design process has come to an end would give them the possibility to improve the operational life of the device as well as its efficiency. It would also result in a more economic and competitive device on the market.

Key-words: CFD, PD-pumps, cavitation modelling

\section{INTRODUCTION}

Positive Displacement (PD) reciprocating pumps are devices utilised in all engineering applications where a fixed mass flow per time unit has to be delivered. In fact, during the pumping cycle, a PD pump delivers the same amount of flow regardless the delivery pressure, the pressure-head curve which is a very important design specification for centrifugal pump, is meaningless for a PD pump. Despite this important feature over the last few decades PD pumps have lost their appeal with respect to their centrifugal counterpart because of the significant design and engineering improvement that the latter have gained. This resulted in a knowledge gap between centrifugal pumps and PD pumps (2) for which it may be said that their design has remained almost unchanged. The numerical analysis methods which have been used to design centrifugal pumps could not be utilised for PD pumps because of their complexity which is not well understood. Cheap and accurate Computational Fluid Dynamics (CFD) methods such as the Multiple Reference Frame (MRF) analysis (3) nowadays are applied to improve the design of centrifugal pumps increasing the gap knowledge between the two kinds of devices. The project behind this paper aims at developing a CFD based analysis tools sufficiently advanced for analysing accurately PD pumps. It is important to point out that no simplified CFD tool for PD pumps exists, such a tool must be capable of simulating the dynamics of the moving parts of the pump such as the valves and the plunger, and the two-way coupling (4) between the valve dynamics and the pressure field. Furthermore, the CFD approach applied in the valve shape optimization, discussed later on in this paper, must be equipped with advanced cavitation models such as the Singhal et al. (1) to account for the cavitating effects in the worst operating conditions the analyst may think of. The proposed comprehensive simulation of a PD pump which has not been previously published in the free technical literature will finally provide engineers with a cheap and reliable tool to review the design of PD pumps in order to:

- Assess the influence of design parameters and specifications on the pump performance in all of the operating conditions.

- Optimize the design of the pump to maximise the operational life. This will result in money saving for the user and an increased visibility on the market for the manufacturer.

The work presented in this paper utilised a numerical approach (Computational Fluid Dynamics, CFD) to test a set of different geometries of valves and seats and collected detailed information of their behaviour in full cavitating conditions to understand the most effective design parameters acting on cavitation and to choose the best design which mitigates, as much as possible, the amount of vapour generated during cavitation. 
The discussed optimization process has to be considered by the reader as an example of application of the CFD model proposed.

\section{TEST CASES DESCRIPTION}

Making use of the ANSYS-Fluent CFD code, two different sensitivity numerical tests were carried out. The first is presented in section 2.1 and deals with the investigation of different valve geometries on the pump performance in cavitating conditions. The second which will be discussed in section 2.2, deals with the investigation of the influence of different valve spring preloads on cavitation. The simulations utilised one pump model running at the same operating conditions, therefore the models differed by the sole valve geometry and spring preload respectively. The pump geometry is shown in Figure 1 which also summarises the initial and boundary conditions. In a similar manner explained by Iannetti (4), the transient CFD model utilised in the present work simulated the inlet stroke. In this first stage of the pumping cycle (Figure 1) the plunger moved backwards sweeping the displacement volume (Figure 2, left), decompressing the pump chamber in order for the pressure forces around the valve to exceed the spring preload then, when the valve lifted off the seat, to suck the working fluid from the inlet manifold and move it into the pump chamber. The initial fluid volume accounted for a zero valve lift and for the plunger located on the Top Dead Centre (TDC) position. A layering (5) moving mesh algorithm managed the volume mesh modification which followed the reciprocating law of the motion generated in the CFD solver by means of the reciprocating motion parameters (shaft angular speed and phase, shaft diameter, connecting rod length). In the test cases where the inlet valve did not finish its motion before the plunger arrived to the bottom Dead Centre (BDC), the initial part of the outlet stroke was also simulated. All of the simulations terminated as soon as the inlet valve returned to the seat again after a delay which depended on the configuration under investigation.

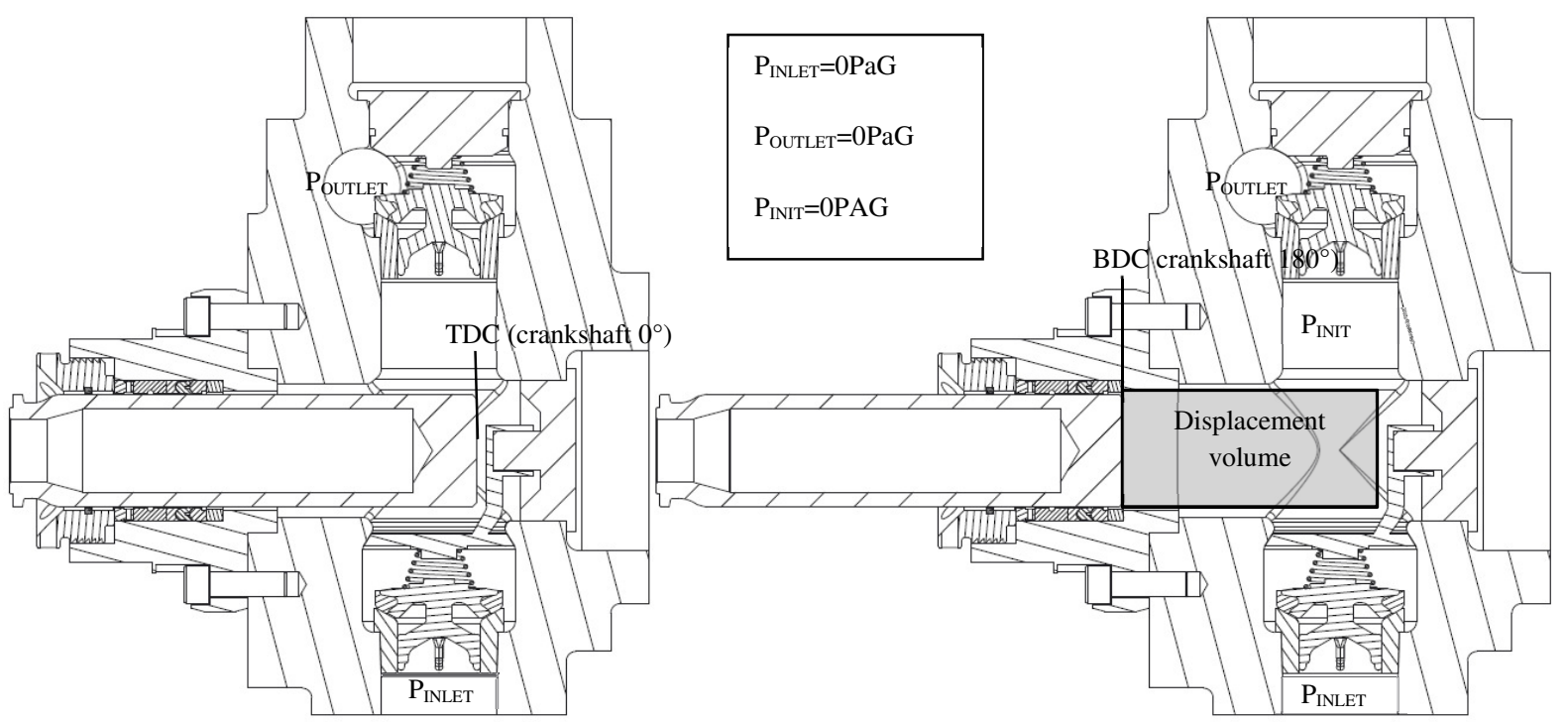

Figure 1: Initial (left) and final (right) configuration of the pump performing the inlet stroke, the starting and ending time of the CFD simulation
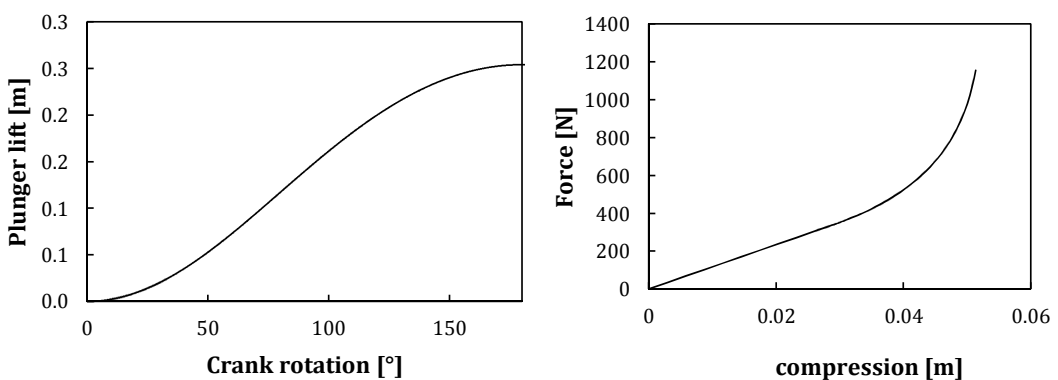

Figure 2: (Left) plunger displacement which was fed into the CFD solver. (Right) spring stress/strain characteristic fed into the UDF. 
The inlet valve fluid volume vicinity was modelled to allow the growth of the valve/seat gap volume which simulated the valve lift and to keep an unchanged geometry of the valve external surfaces during the valve motion, this is explained in Figure 4.

The valve dynamics, which the CFD model accounted for, were the result of the integration of the overall forces. The spring force-compression characteristic curve (spring rate diagram of Figure 2, right) was fed into the User Defined Function (UDF) via a polynomial law and the preload was set fixing the spring compression at zero lift which is usually a design parameter, the diagram of Figure 5 shows how the UDF interfaces with the main CFD solver. The initial gauge pressure in all of the volumes was set to $0 \mathrm{~Pa}$ while the inlet pressure accounted for the inlet manifold pressure drop at non-zero mass flow as explained by Iannetti (4) via a mass flow dependent pressure inlet condition.

The "full" cavitation model developed by Singhal (1) was utilised to drive the liquid-vapour phase transformation and a four transport equation multiphase model (Mixture model, (3)) was chosen for the Raynolds Averaged Navier-Stokes (RANS) solver. The Singhal et al. cavitation model is an advanced model because it accounts for all the first order terms of the Rayleigh (6) equation and also for the surface tension forces term and non-condensable gas effect added by Plesset (7). As already discussed in (4) the model simulated the valve lift making use of the layering moving mesh technique (5) which was also utilised for the plunger displacement simulation. The work process is summarised in the following paragraph.
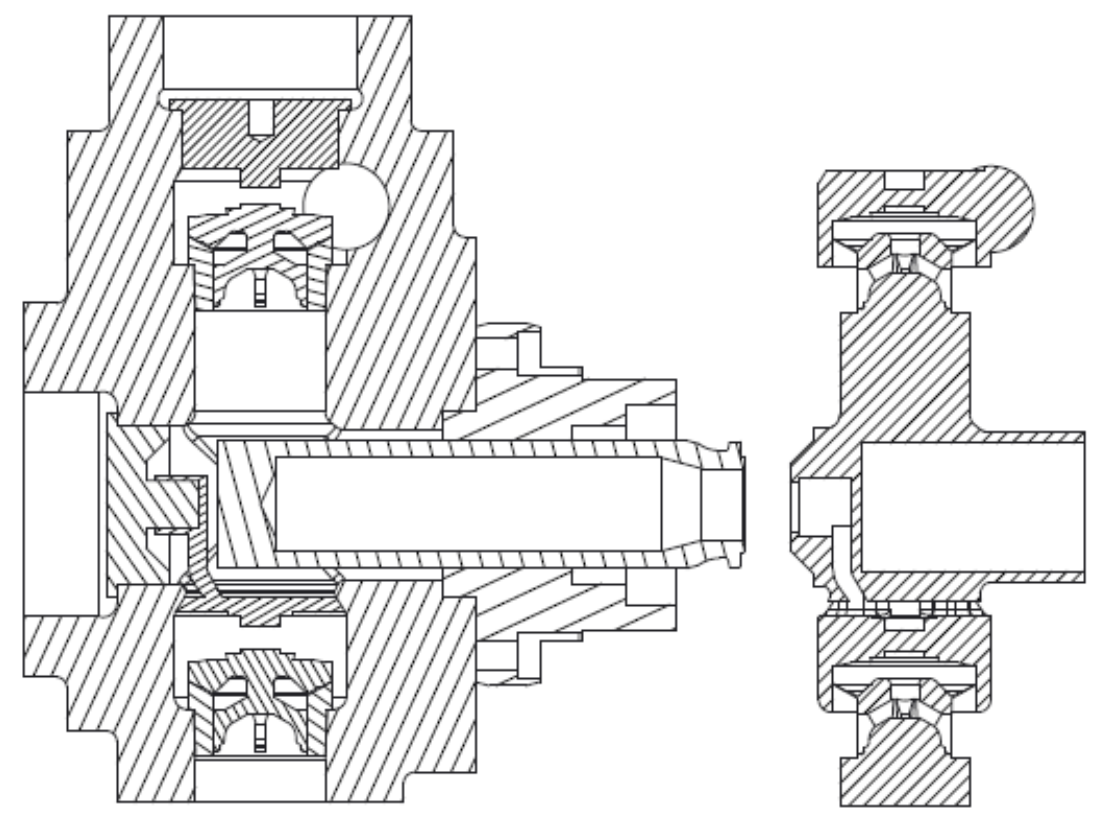

Figure 3: Solid to fluid volumes, Boolean operation.

Volume and mesh preparation

The fluid volumes were extracted from the solid geometry (Figure 3) with the plunger positioned at the TDC and the valves on the seat (configuration at initial time) by means of Boolean operation. The fluid volume was decomposed in the pattern shown in Figure 4 to accommodate for the moving mesh technique. For the displacement volume which was a cylindrical volume located on the top of the plunger surface, a full hexahedral mesh was chosen, the mesh layering algorithm created parallel layers of hexahedral cells while the plunger moved backwards for the inlet stroke and destroyed them during the discharge stroke, the rate of layers creation/destruction was governed by the "In-cylinder" technique (5) which turned the reciprocating parameters provided (shaft angular speed and phase, crank diameter, connecting rod length) into the plunger displacement and velocity at every time step. 


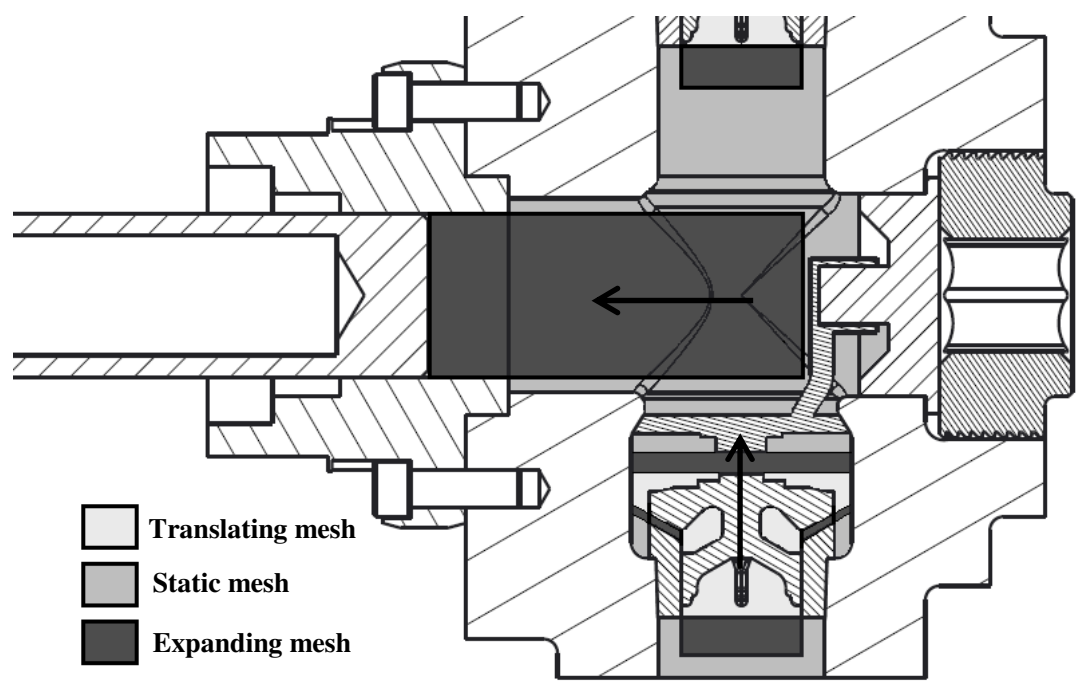

Expanding mesh

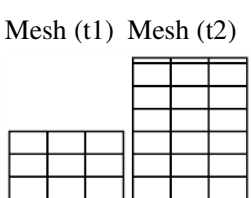

EXPANDING

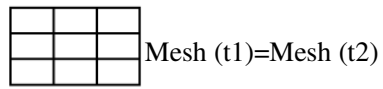

STATIC

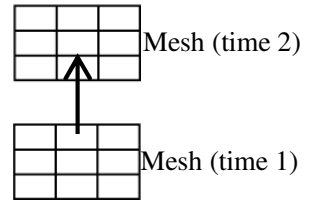

TRANSLATING

Figure 4: Fluid volume decomposition pattern to accommodate for the moving mesh technique (8)

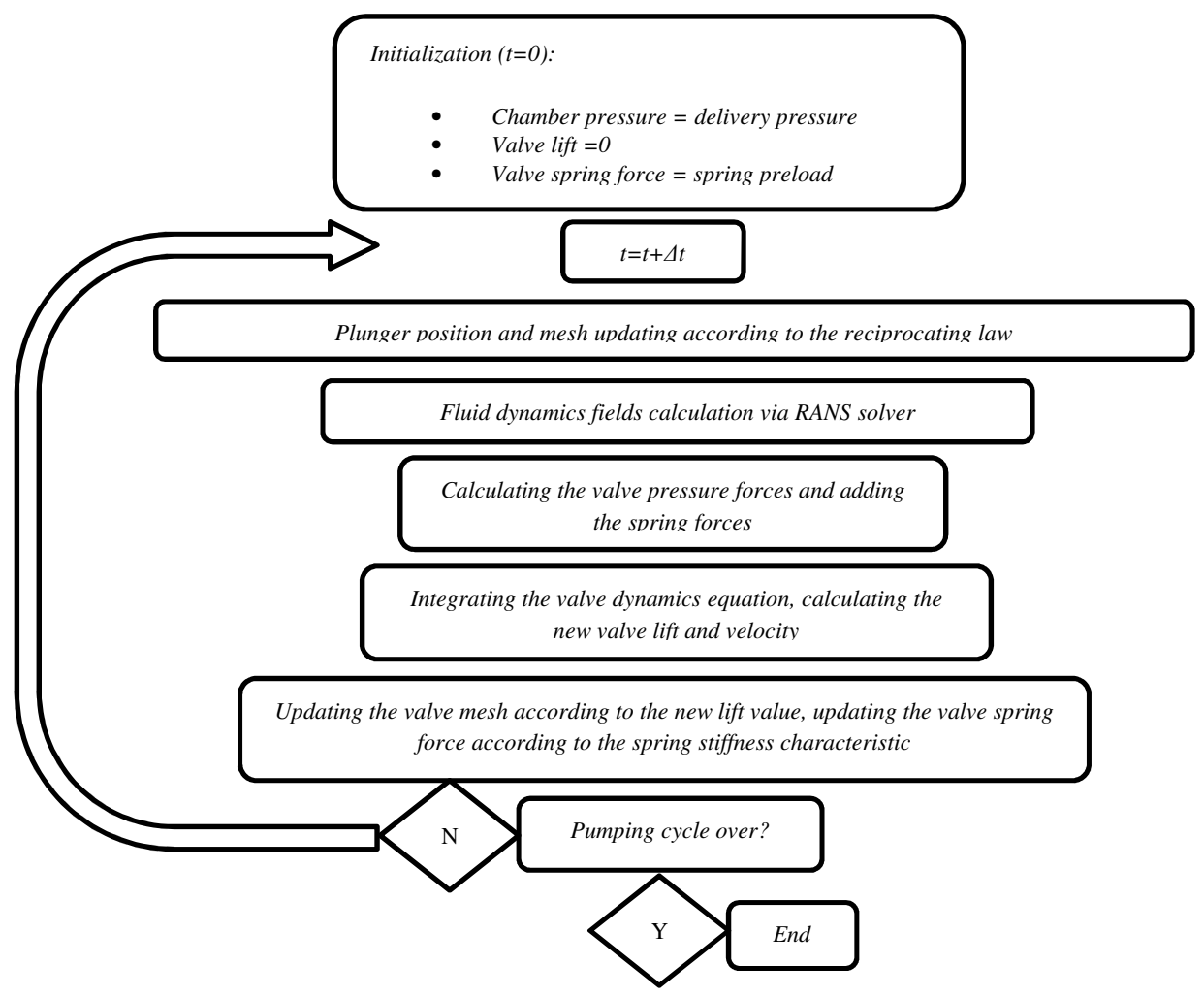

Figure 5: User Defined Function to manage the two-way coupling (pressure field-valve lift). How it relates to the CFD main solver.

The valve lift was simulated in a similar way. The fluid volume around the valves was decomposed in order to obtain cylindrical volumes on the top and bottom. When the valve lifted up, the upper volume compressed destroying hexahedral cell layers while the bottom volume expanded adding cell layers. Vice versa while the valve sit down. Similarly the valve-seat gap volume was modelled as an annular shaped volume which expanded when the valve lifted off and compressed when sit down. The volumes in between the compressing/expanding region were meshed using tetrahedral cells and were subjected to a rigid motion of translation which followed the layers creation/destruction. The algorithm governing every time step the valve lift and velocity was a $\mathrm{C}++$ User Defined Function (UDF) which calculated the overall valve force integrating it to calculate valve velocity and displacement. The overall forces were calculated adding the spring forces to the pressure forces (calculated in the previous time step). The UDF dynamically related the solution of the Reynolds Averaged Navier Stokes (RANS) solver to the mesh motion. The valve lift resulted self-actuated, no external action by the analyst was needed. 
The compressibility of the liquid was also modelled. This is not an option in cases of very high pressure or when the valves are both closed (e.g. beginning of the suction stroke) and a moving plunger would not allow the achievement of the mass continuity if the liquid was not compressible. As the compressibility of water is very low, an explicit model was utilised (8) so that the pressure field affected the density but the latter did not affect the former.

A mesh sensitivity analysis was carried out to define the best mesh spacing to deal with the needs of good accuracy and low computational efforts. Three meshes were tested; 3, 5 and 6 million cells and according to the results the 5 million cells was utilised because it proved the best computational performance and good accuracy.

\section{Solver settings}

A k- $\varepsilon$ turbulence model was chosen because it proved better convergence behaviour over other models such as $\mathrm{k}-\omega$ and, to solve the problem of low $\mathrm{y}^{+}$(null minimum velocity) the "enhanced wall treatment" (3) was utilised as near wall algorithm. The enhanced wall treatment corrects the standard wall function in cases when the commonly accepted maximum value of $\mathrm{y}^{+}$of 100 is not achieved. A two equations multiphase model (the mixture model (3)) was chosen to deal with the two phases (water and vapour) which also accounted for a little amount of non-condensable gas, treated as ideal gas. The already mentioned Singhal et al. cavitation model was utilised to handle the phase change. Table 1 summarises the settings chosen.

Table 1: Summary of the solver settings (4)

\begin{tabular}{|c|c|c|c|c|c|}
\hline \multicolumn{2}{|l|}{ Solver } & \multicolumn{4}{|c|}{ RANS, pressure based, transient } \\
\hline \multirow{5}{*}{ Models } & \multirow{3}{*}{ Multiphase } & \multicolumn{4}{|c|}{ Mixture model (3) } \\
\hline & & \multirow{2}{*}{ Phases } & \multicolumn{2}{|c|}{ Water liquid } & Primary phase \\
\hline & & & \multicolumn{2}{|c|}{ Water vapour } & Secondary phase \\
\hline & Turbulence & $\mathrm{K}-\varepsilon$ Standard & \multicolumn{3}{|c|}{ Enhanced wall treatment } \\
\hline & Cavitation & Singhal et al. & \multicolumn{3}{|c|}{15 ppm air (ideal gas) } \\
\hline \multicolumn{2}{|c|}{ Pressure-Velocity coupling } & \multicolumn{4}{|l|}{ SIMPLE } \\
\hline \multirow{4}{*}{\multicolumn{2}{|c|}{ Spatial discretization }} & \multicolumn{2}{|l|}{ Momentum } & \multicolumn{2}{|c|}{ Second order upwind } \\
\hline & & \multicolumn{2}{|l|}{ Vapour } & \multicolumn{2}{|c|}{ First order upwind } \\
\hline & & \multicolumn{2}{|c|}{ Turbulent kinetic energy } & \multicolumn{2}{|c|}{ Second order upwind } \\
\hline & & \multicolumn{2}{|c|}{ Turbulent dissipation rate } & \multicolumn{2}{|c|}{ Second order upwind } \\
\hline \multicolumn{2}{|c|}{ Transient formulation } & \multicolumn{4}{|c|}{ First order implicit } \\
\hline \multirow{5}{*}{\multicolumn{2}{|c|}{ Under relaxation factors }} & \multicolumn{2}{|l|}{ Pressure } & \multicolumn{2}{|l|}{0.3} \\
\hline & & \multicolumn{2}{|l|}{ Momentum } & \multicolumn{2}{|l|}{0.7} \\
\hline & & \multicolumn{2}{|l|}{ Vapour } & \multicolumn{2}{|l|}{0.5} \\
\hline & & \multicolumn{2}{|c|}{ Turbulent kinetic energy } & \multicolumn{2}{|l|}{0.8} \\
\hline & & \multicolumn{2}{|c|}{ Turbulent dissipation rate } & \multicolumn{2}{|l|}{0.8} \\
\hline Residua & & $10^{-3}$ & & & \\
\hline Time ste & & $0.125^{\circ}$ crank $\mathrm{r}$ & $n \equiv 1$ & $10^{-4} \mathrm{~s}$ & $130 \mathrm{rpm}$ \\
\hline Max Iter & e step & 35 & & & \\
\hline & & Compressibilit & ater $(8$ & & \\
\hline UDFs & & Valve dynamic & ure 5 & & \\
\hline
\end{tabular}

A 12 GB RAM computer with an Intel Xeon W3670 @3.2 GHz 6 core processor was employed for the each simulation presented which needed roughly 2 weeks to accomplish half a pumping cycle (inlet stroke only).

\section{$2.1 \quad$ Geometry sensitivity tests}

A set of five valve/seat modified configurations were considered for the analysis. Figure 6 represents the original geometry of which Figure 7 to Figure 11 are proposed modifications. The original geometry is a common valve which slides inside the seat inner $83 \mathrm{~mm}$ diameter bore by means of four legs which allow the translation along the vertical axis only. 

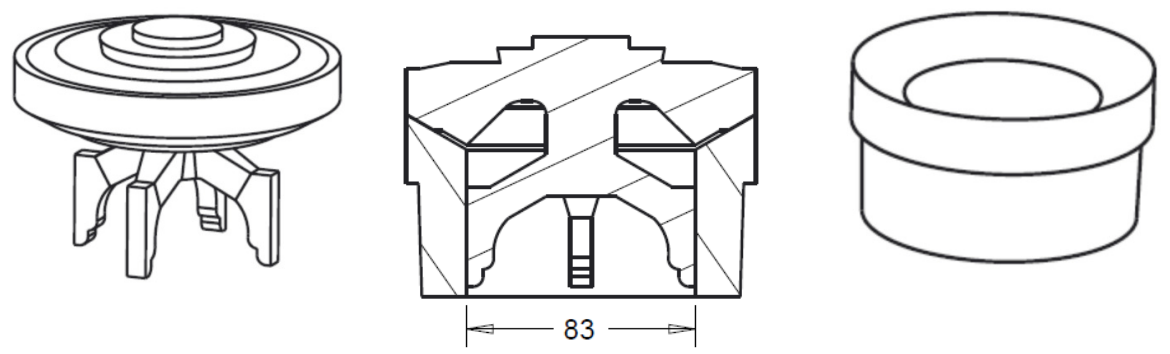

Figure 6: Initial valve/seat configuration

The first tested modification (Mod1) of the valve/seat configuration is represented in Figure 7. A stem located on the top of the valve replaced the four leg of the original configuration. The stem was designed to slide in a bore drilled in the inlet valve spring retainer (visible in Figure 1). The seat inner diameter was increased to 100 $\mathrm{mm}$.
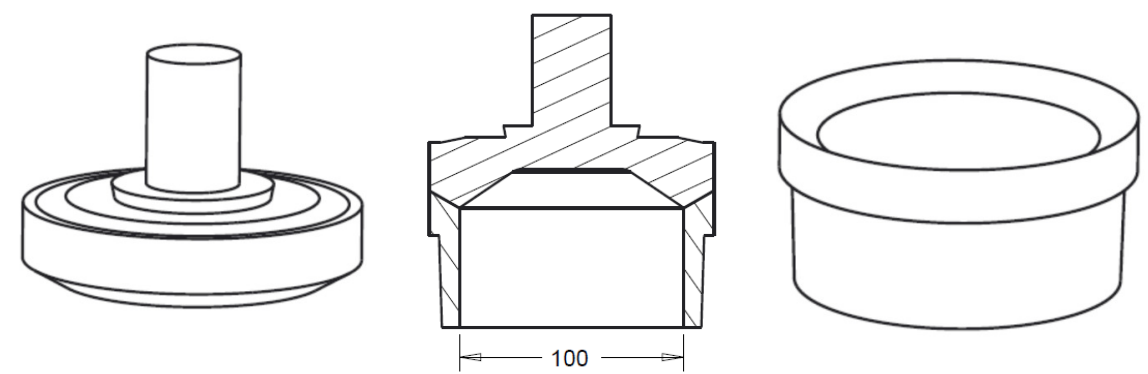

Figure 7: Modification number 1 design

The design modification number 2 (Mod2) is represented in Figure 8, it shows a stem valve with a shorter seat of $110 \mathrm{~mm}$ of inner diameter, a flat bottom valve surface may be observed.
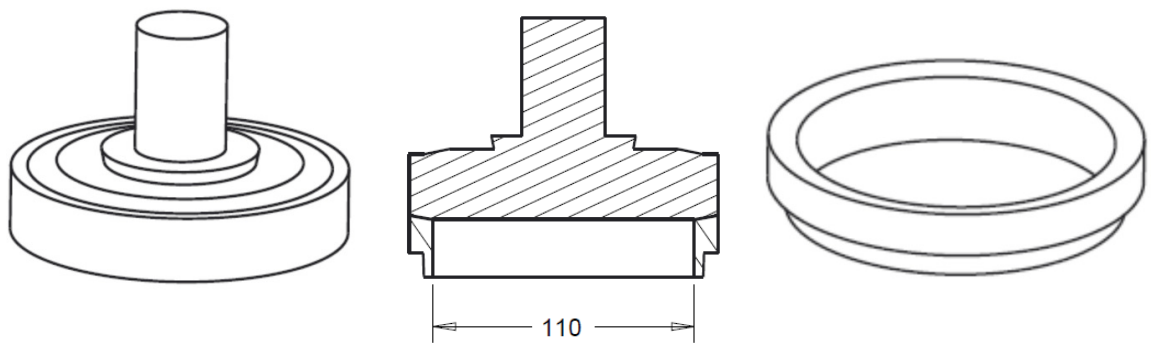

Figure 8: Modification number 2 design

The modification number 3 (Mod3) is showed in Figure 9. In this case the seat inner diameter was unchanged but three additional opening areas were cut in the valve main body, an additional annular body in the seat was needed to provide the additional openings with a contact area to close them at zero valve lift situations. The idea being verified was the increment of the the overall flowing area to bring down the pressure drop across the valve. 


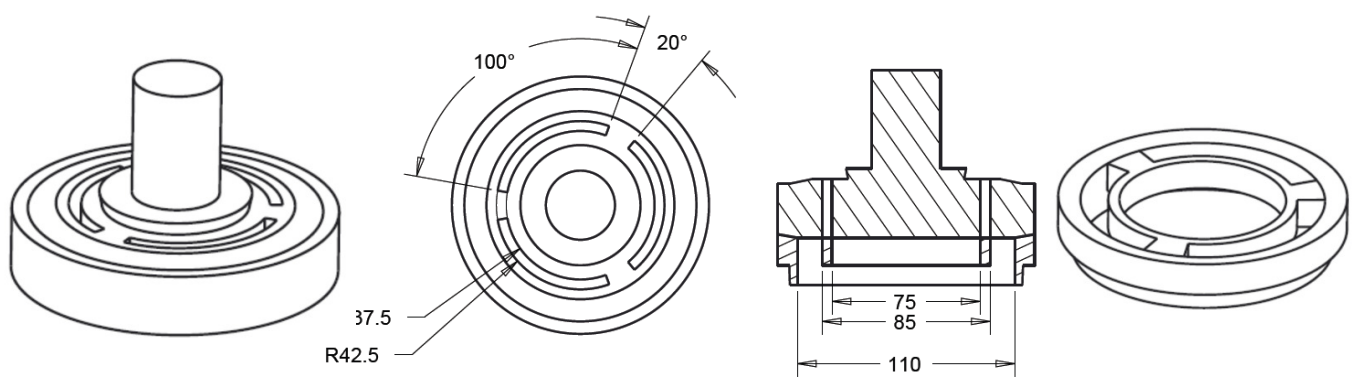

Figure 9: Modification number 3 design

The modification number 4 (Mod4) is represented in Figure 10, in this case, unlike the previous figure one circular additional opening area was chosen and cut in the stem. An additional body was designed in the seat to seal the flow.
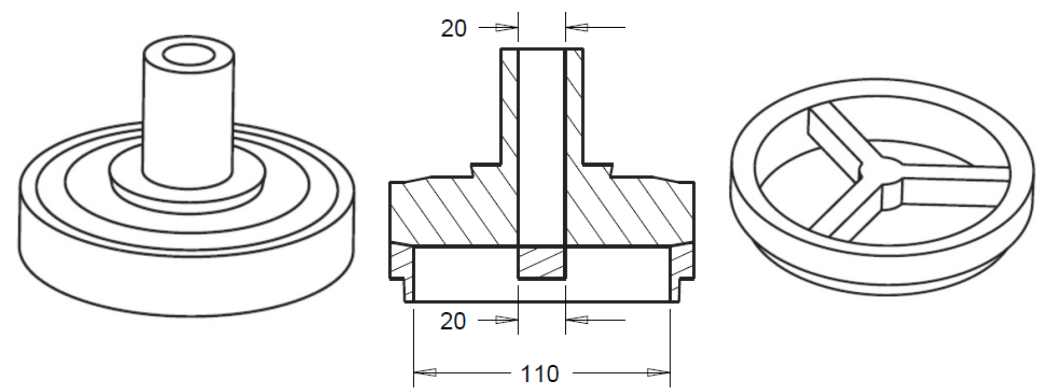

Figure 10: Modification number 4 design

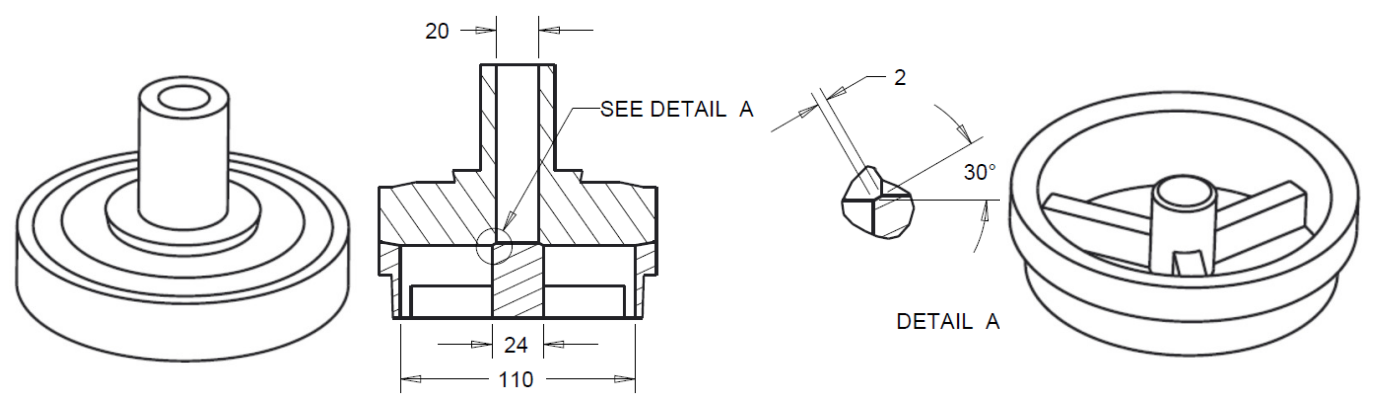

Figure 11: Modification number 5 design

The last configuration (Mod5) proposed and tested is shown in Figure 11. It differs from the previous figure by detail A. The seat additional contact body was enlarged and its angle with the valve was set to $30^{\circ}$. The interest in testing this little variation was suggested by the need to estimate the sensitivity of the results when the design moves to a more feasible configuration. Mod3 and Mod4, in fact were characterised by the same additional contact/opening surfaces which did not provide sufficient sealing properties that would be required in real designs. Mod3 and Mod 4 were tested to verify the physical principle rather than providing with a feasible geometry. 


\subsection{Spring preload sensitivity test}

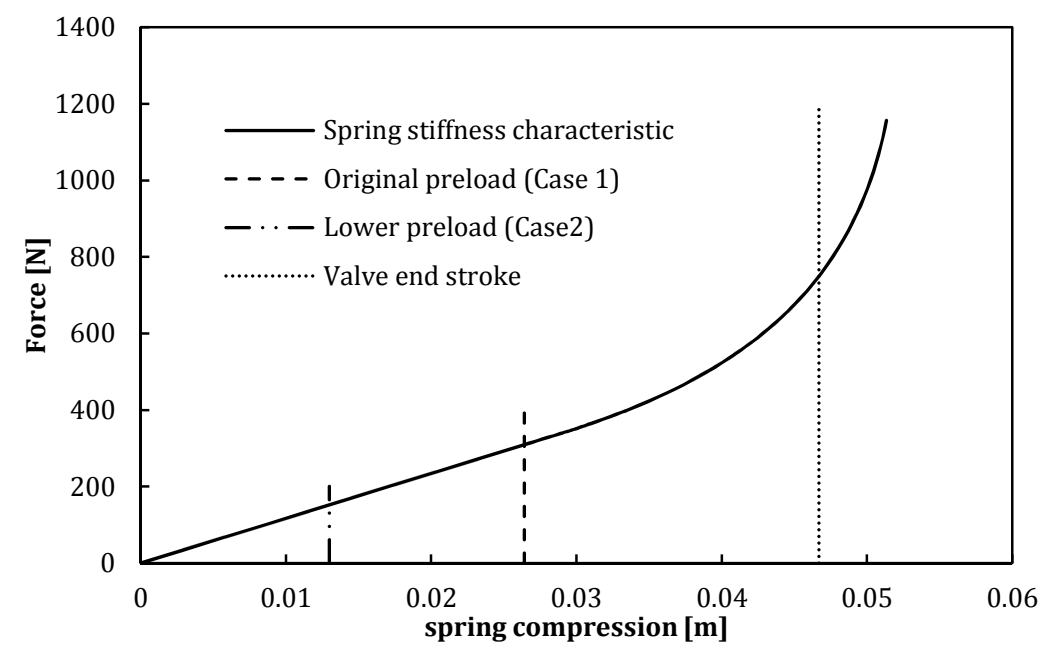

Figure 12: Valve stiffness characteristic and chosen preload for the 2 cases discussed

Figure 12 shows in more details the stiffness characteristic of the spring which was fed into the UDF. The pump model with the original valve-seat geometry configuration was simulated in two cases. In the first case which was the original, the spring preload was defined by fixing the spring compression at closed position at $26 \mathrm{~mm}$ and that gave an initial preload of $300 \mathrm{~N}$ circa, in the second case the spring was compressed of $13 \mathrm{~mm}$ at zero lift and this gave a preload of $150 \mathrm{~N}$ circa. The objective in this second analysis was to obtain higher valve lift under similar external pressure forces and assess how much this affects the vapour generation by comparison with the former case.

\section{NUMERICAL RESULTS DISCUSSION}

To compare the six geometries presented in section 2.1 or the two different operating conditions of section 2.2 . The following sets of monitor were chosen:

- Chamber pressure. The static gauge pressure in a chosen static monitor point located in the vicinity of the TDC position of the plunger.

- Valve lift. Displacement of the valve throughout the induction stroke.

- Overall flowing area. To compare the geometries behaviour the overall valve open area was calculated/monitored as the sum of the main open area between the valve and the valve seat and the additional area created by extra openings in the valve body or the valve stem where present. Both depended on the valve lift.

- Pump Chamber vapour volume integral. The overall amount of vapour created was monitored as evidence which summarised the effect of the changes in valve geometry and spring preload on the presence of cavitation.

All this transient quantities were plotted in the domain of the crank angular rotation $\left(0^{\circ}-180^{\circ}\right)$ instead of time as this provides the reader with a better understanding of the phenomena.

\subsection{Valve shape sensitivity test results}

Figure 13 shows the chamber pressure comparison between the six configurations throughout the induction stroke. The figure demonstrates clearly that moving from the original valve-seat shape to Mod2 a significant increment of the minimum chamber pressure was achieved. While the original shape pressure approached closely the vapour pressure, Mod1 which was characterised by a bigger seat inner diameter, showed a higher pressure. Figure 13 identifies Mod2 as the design showing the highest safety factor against cavitation as the pressure line remains above all the counterparts. Furthermore, Mod3 to Mod5 configurations showed similar trends as the minimum pressure achieved was similar for all of them. Figure 14 shows the valve lifts trends, the highest maximum lift was achieved by the Mod2 configuration which also showed (Figure 15) the widest flowing 
area, this explains the reason why Mod2 reveals the maximum chamber peak pressure. Mod3 showed the least valve lift but the second widest maximum flowing area. This can be explained by taking into account the additional opening area which increases the flowing area per valve lift unity. On average it is clear that in Mod3, 4 and 5 the cuts in the main valve body designed to create the additional opening areas decreased the valve overall surface over which the pressure force operates and caused a decrement of the valve lift force and thus maximum valve lift. This produced a reduction in the main flowing area which was just partially covered by the additional opening area in the valve body. Even though Mod3 showed the highest gain in overall flowing area it was not sufficient to overcome and improve on the Mod2 trend. Analysis of the Mod1 results revealed an unexpected behaviour. It showed the second highest maximum lift (Figure 14) and overall flowing area (Figure 15) but the pressure trend (Figure 13) revealed the second lowest pressure peak which resulted in the second worse cavitation behaviour. This seems not to agree with the average trend. The reasons for this phenomenon are to be found in the geometric differences between Mod1 and 2 which one can observe in Figure 7 and Figure 8 and will be investigated further. Small variations in the geometry did not result in significant differences in behaviour as demonstrated by Mod5 which showed all the trends of Figure 13 to Figure 16 to be consistent with the Mod4 counterpart.

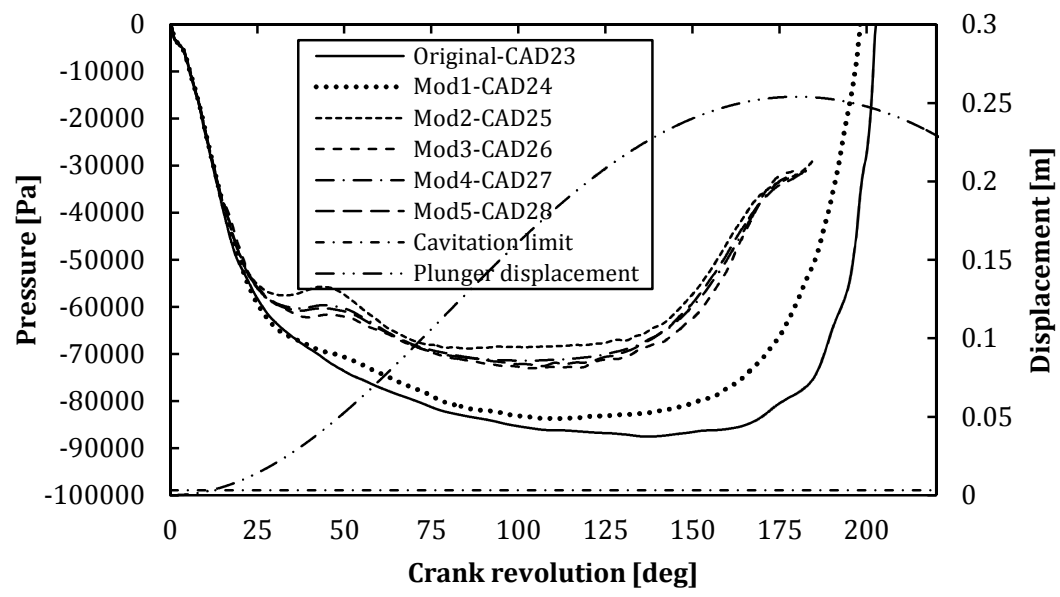

Figure 13: Chamber pressure

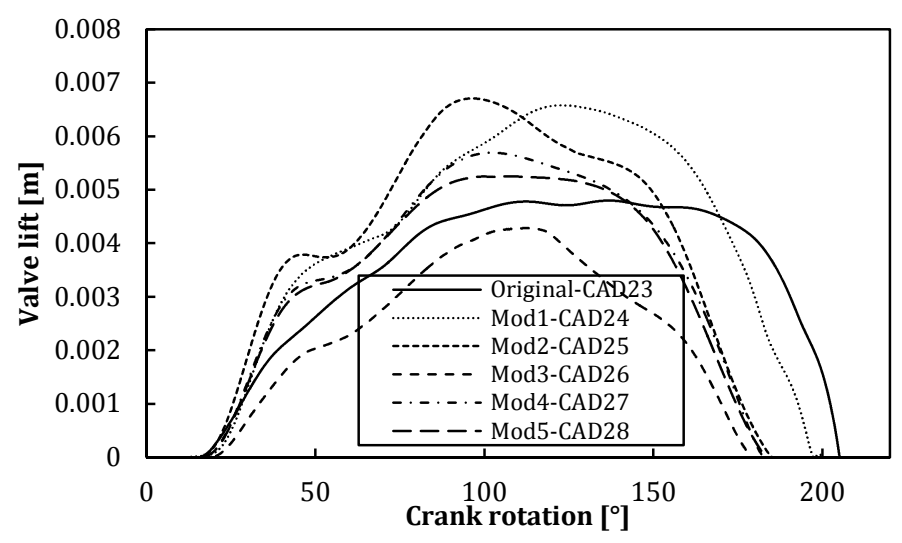

Figure 14: Valve lift 


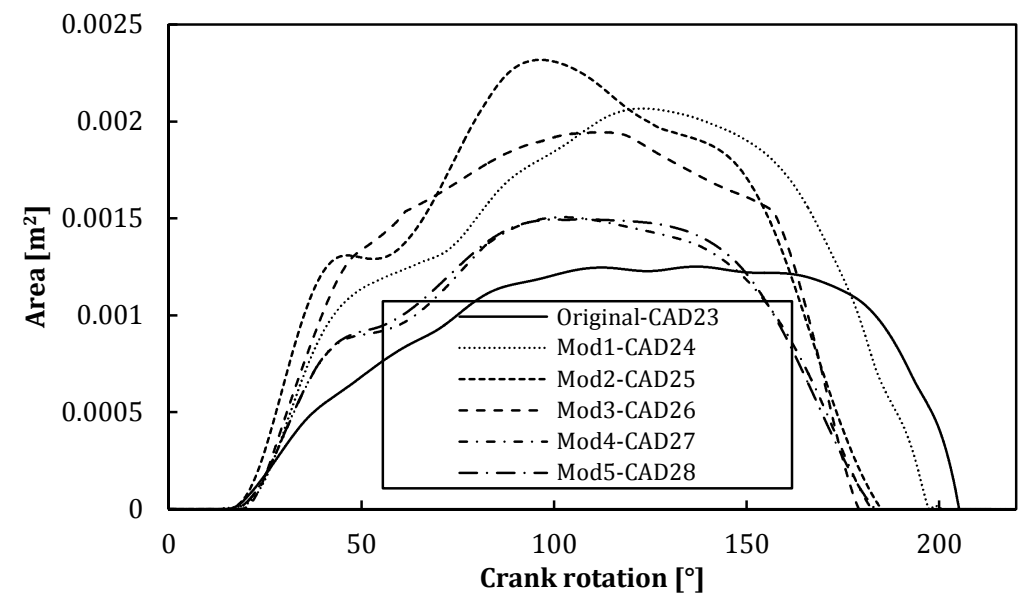

Figure 15: Overall flowing area

Figure 16 shows the chamber vapour volume integral for all of the configurations whereas Table 2 summarizes the most relevant outcomes of the CFD simulations. The pump equipped with the original valve/seat configuration experienced the highest amount of vapour generation. This caused the lowest volumetric efficiency as one can see in Table 2. As expected, Mod2 was the least affected by the vapour generation even though Mod3 to 5 did not show a significantly different trend with respect to Mod2. Table 2 relates the volumetric efficiency with the vapour integral peak of Figure 16 and the inlet valve opening and closing times. The original valve showed the highest delay in opening which was related to the smallest valve area where the pressure drop operated due to the smallest inner valve-seat diameter. Mod1 and 2 which have a larger inlet diameter (100 and $110 \mathrm{~mm}$ respectively) were subjected to a higher lifting force which exceeded the spring preload sooner than the original valve case. From Mod3 to 5 the cuts in the valve body caused a reduction in the pushing area and thus the valve lifted off the seat later than the Mod2 case. Another important remark which Table 2 reveals is the direct link between the volumetric efficiency, the vapour generation and the valve closing time delay. The higher is the pump chamber vapour production the higher is the delay and the lower is the volumetric efficiency.

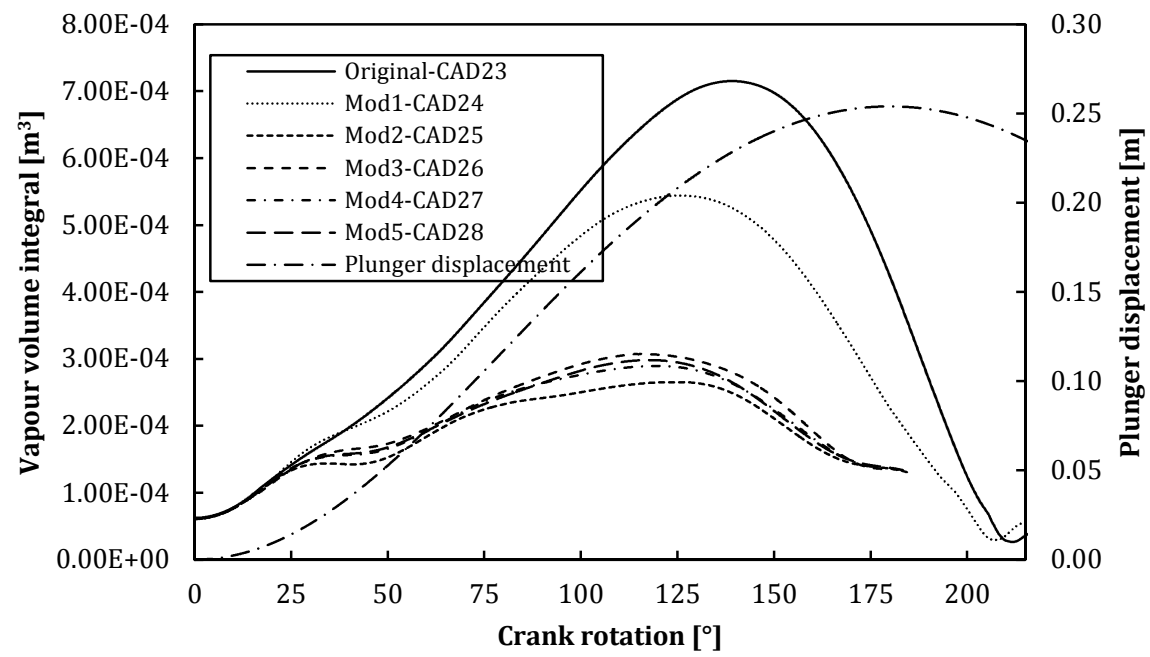

Figure 16: Chamber vapour integral

Table 2: Summary and comparison of the six configurations

\begin{tabular}{lccccc}
\hline & $\begin{array}{c}\text { Volumetric } \\
\text { efficiency } \\
{[\%]}\end{array}$ & $\begin{array}{c}\text { Inlet valve } \\
\text { opening time } \\
{[\%]}\end{array}$ & $\begin{array}{c}\text { Inlet } \\
\text { valve } \\
\text { closing } \\
\text { time }\left[{ }^{\circ}\right]\end{array}$ & $\begin{array}{c}\text { Maximum } \\
\text { chamber vapour } \\
\text { volume } \times \mathbf{1 0}^{-3}\left[\mathbf{m}^{\mathbf{3}}\right]\end{array}$ & $\begin{array}{c}\text { Maximum } \\
\text { Valve-seat gap } \\
\text { vaur volume } \\
\times \mathbf{1 0}^{-3}\left[\mathbf{m}^{\mathbf{3}}\right]\end{array}$ \\
\hline $\begin{array}{l}\text { Original } \\
\text { CAD23 }\end{array}$ & 93.36 & 15.6 & 205.3 & 0.7151 & \\
\hline $\begin{array}{l}\text { Mod 1 } \\
\text { CAD24 }\end{array}$ & 95.1 & 12.25 & 200.75 & 0.5441 & 0.0084577 \\
\hline
\end{tabular}




\begin{tabular}{lccccc}
\hline $\begin{array}{l}\text { Mod 2 } \\
\text { CAD25 }\end{array}$ & 97.07 & 11.74 & 185.06 & 0.2652 & 0.00190485 \\
\hline $\begin{array}{l}\text { Mod 3 } \\
\text { CAD26 }\end{array}$ & 96.78 & 12.5 & 179.12 & 0.3075 & 0.0019380 \\
\hline $\begin{array}{l}\text { Mod 4 } \\
\text { CAD27 }\end{array}$ & 97.04 & 12.0 & 182.62 & 0.2895 & 0.0016658 \\
\hline $\begin{array}{l}\text { Mod 5 } \\
\text { CAD28 }\end{array}$ & 97.01 & 12.0 & 183.37 & 0.2982 & 0.0018992 \\
\hline
\end{tabular}

\subsection{Spring preload sensitivity test results}

As shown in Figure 17 the spring preload affects significantly the chamber pressure, case 2 minimum peak pressure was $10 \mathrm{kPa}$ circa higher than case1. This depended on the higher valve lift achieved by the lower preload case as one can see in Figure 18. The higher lift resulted in an increased flowing area and a consequently lower velocity which caused a lower pressure drop across the valve. Figure 19 and Table 3 complete the overview of this analysis showing the amount of vapour generated in the pump chamber and correlate the delay in opening and closing time with the vapour generation. A decrement in the spring preload results in a smaller delay of the valve opening time. Furthermore, the higher lift caused a lower amount of vapour generation which lowered the closing delay. As explained by Iannetti (4) the pump utilised the initial part of the outlet stroke to compress the vapour and turn it into water at constant pressure (vapour pressure), therefore a lower amount of vapour means usually a smaller delay because the compressing process lasts for a shorter period of time.

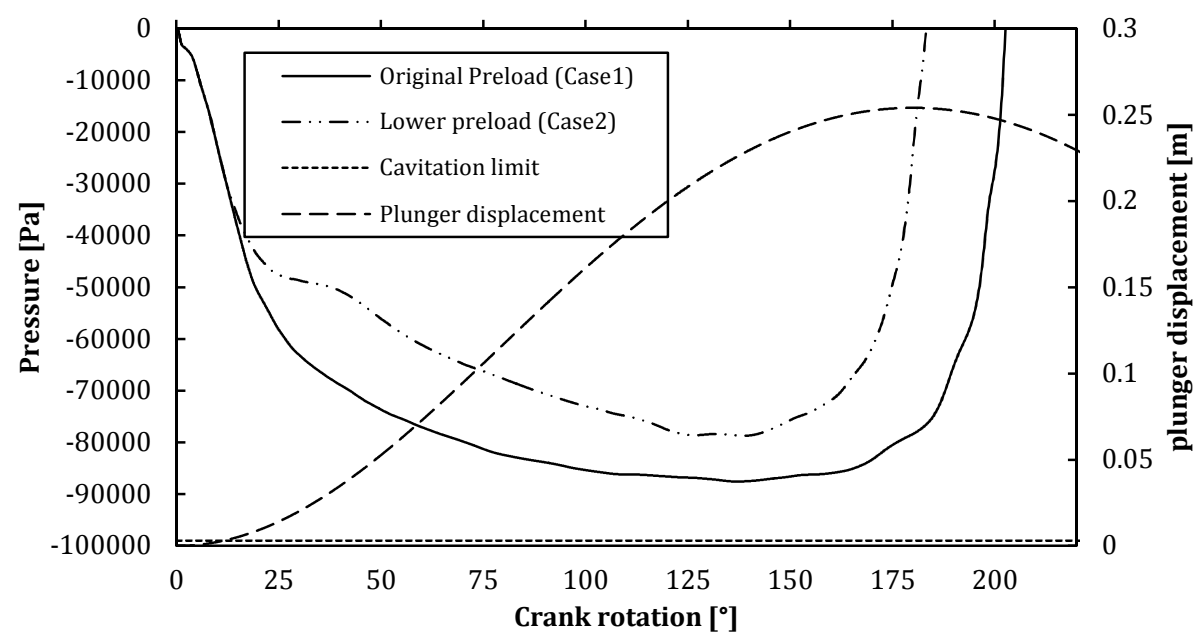

Figure 17: Chamber pressure history original and lower preload spring case on the original valve-seat geometry 


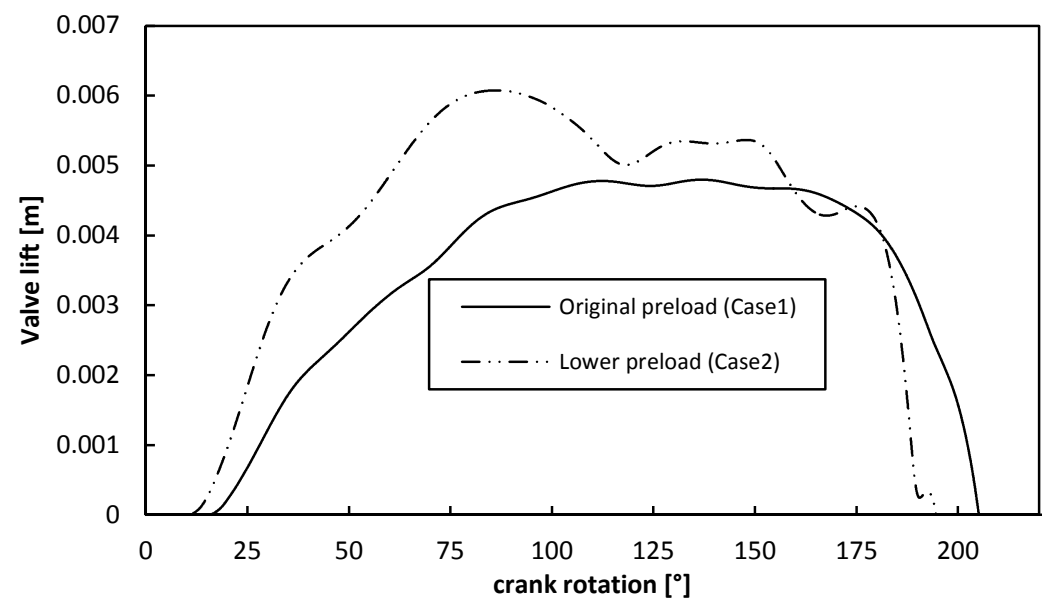

Figure 18: Valve lift-time histories. Case 2 shows a higher maximum lift and as a consequence, a wider flowing area.

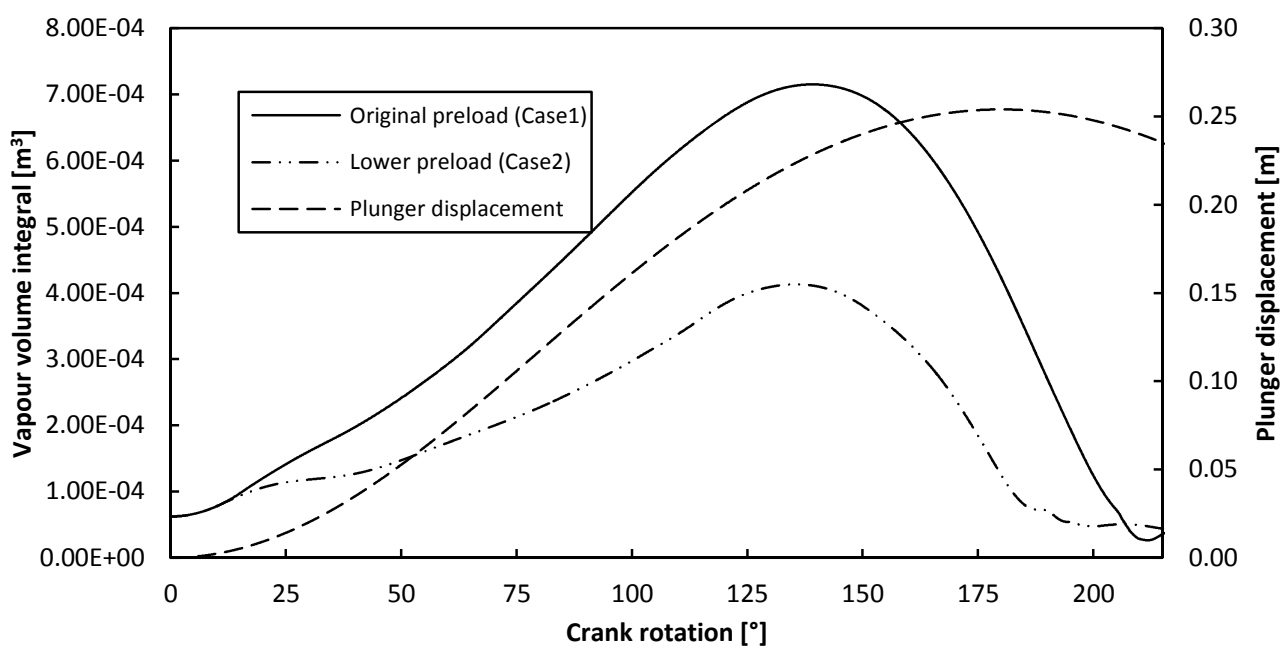

Figure 19: Pump chamber vapour integral throughout the inlet stroke, Case 2 showed a significant reduction in vapour generation.

Table 3: Summary of the spring preload sensitivity test. Case 2 shows a lower valve opening and closing delay and higher efficiency

\begin{tabular}{ccccc}
\hline & $\begin{array}{c}\text { Volumetric } \\
\text { efficiency } \\
{[\%]}\end{array}$ & $\begin{array}{c}\text { Inlet valve } \\
\text { opening time } \\
{[\%]}\end{array}$ & $\begin{array}{c}\text { Inlet valve } \\
\text { closing time } \\
{\left[{ }^{\circ}\right]}\end{array}$ & $\begin{array}{c}\text { Maximum } \\
\text { chamber } \\
\text { vapour volume } \\
\times \mathbf{1 0}^{-3}\left[\mathbf{m}^{\mathbf{3}}\right]\end{array}$ \\
\hline $\begin{array}{c}\text { Valve original } \\
\text { preload (Case1) }\end{array}$ & 93.36 & 15.6 & 205.12 & 0.7151 \\
\hline $\begin{array}{c}\text { Lower spring } \\
\text { preload (case2) }\end{array}$ & 98.54 & 10.25 & 194.75 & 0.4129 \\
\hline
\end{tabular}

\section{CONCLUSION}

Two separate CFD analyse of the same PD reciprocating pump working at the same operating conditions were carried out.

The first analysis was aimed at verifying which one among five modified valve and valve seat geometries of the original design was the best to mitigate vapour generation in full cavitating conditions. The analysis identified the Mod2 as the best configuration but, more importantly, it revealed the geometric parameter that affects the 
cavitation phenomenon the most which is the valve and valve seat diameter. It also showed the sensitivity of the geometry modification on the phenomenon under investigation. The model showed reliable results and demonstrated the potential of the CFD approach on design optimization.

The second analysis was aimed at testing the PD pump equipped with the original valve and valve seat under $300 \mathrm{~N}$ and $150 \mathrm{~N}$ of inlet valve spring preload respectively by adjusting the compression of the valve at closed position according to the trend of Figure 12. A high spring preload is usually beneficial in non-cavitating conditions because it accelerates the valve closing process when the inlet stroke has come to the end and minimizing the slip during this process. This second analysis revealed that a high spring preload is not beneficial in cavitating condition because it increases the vapour generated.

\section{REFERENCE LIST}

1. Singhal AK, Athavale MM, Li H, Jiang Y. Mathematical Basis and Validation of the Full Cavitation Model. J Fluids Eng. Asme-Amer Soc Mechanical Eng; 2002;124(3):617.

2. Tackett HH, Cripe JA, Dyson G. Positive displacement reciprocating pump fundamentals- power and direct acting types. Proceedings of the twenty-fourth international pump user symposium. Texas A\&M University; 2008. p. 45-58.

3. ANSYS. ANSYS Fluent Theory Guide. ANSYS Fluent; 2011.

4. Iannetti A, Stickland M, Dempster W. A CFD study on the mechanisms which cause cavitation in positive displacement reciprocating pumps. Proc Inst Mech Eng Part A J power energy. 2014;In Press.

5. $\quad$ ANSYS. ANSYS FLUENT User's Guide. ANSYS Inc.; 2011.

6. Rayleigh, Lord. VIII. On the pressure developed in a liquid during the collapse of a spherical cavity. Philos Mag Ser 6. Series 6, . 1917 Aug;34(200):94-8.

7. Plesset MS, Prosperetti A. BUBBLE DYNAMICS AND CAVITATION. Annu Rev. 1977;9:145-85.

8. Iannetti a., Stickland MT, Dempster WM. A computational fluid dynamics model to evaluate the inlet stroke performance of a positive displacement reciprocating plunger pump. Proc Inst Mech Eng Part A J Power Energy. 2014 Apr 7;228(5):574-84. 